\title{
The HuoXue DiTan Recipe Attenuates Left Ventricular Hypertrophy in Spontaneously Hypertensive Rats by Increasing Autophagy Through the PTEN/PI3K/AKT/mTOR Pathway
}

\section{YAO Jiamei}

Xiangya Hospital Central South University

ZHANG Cui

Xiangya Hospital Central South University

YANG Yushu

Xiangya Hospital Central South University

\section{YANG Haiyan}

Xiangya Hospital Central South University

ZHANG Dan

Xiangya Hospital Central South University

SHI Yongyue

Xiangya Hospital Central South University

CHEN Qiong

Xiangya Hospital Central South University

Guangwei Zhong ( $\sim$ zgw7512@sina.com )

Xiangya Hospital Central South University https://orcid.org/0000-0001-5844-4171

\section{Research}

Keywords: HuoXue DiTan recipe, left ventricular hypertrophy, hypertension, autophagy, PTEN/PI3K/AKT/mTOR pathway

Posted Date: November 23rd, 2021

DOI: https://doi.org/10.21203/rs.3.rs-1047223/v1

License: (9) This work is licensed under a Creative Commons Attribution 4.0 International License. Read Full License 


\section{Abstract \\ Background}

Hypertension-induced left ventricular hypertrophy (LVH) is associated with a reduction in autophagy, which can be inhibited by disruption of the PTEN/PI3K/AKT/mTOR pathway. The HuoXue DiTan recipe (HDR) is a commonly used prescription that has shown therapeutic effects on hypertension and its complications. However, its mechanisms are still unclear. In the present study, we hypothesized that HDR can regulate the PTEN/PI3K/AKT/mTOR signaling pathway and thereby reverse LVH by increasing autophagy in spontaneously hypertensive rats.

\section{Methods}

Twelve-week-old male spontaneously hypertensive (SH) rats and age-matched normotensive-control Wistar-Kyoto (WKY) rats were divided into four groups. After 12 weeks of treatment, echocardiographic measurements were made on the left ventricle, blood samples were collected for oxidative stress analysis, left ventricle tissue was processed for hematoxylin and eosin, Masson's trichrome, and immunohistochemical/ immunofluorescence staining, and TUNEL, RT-qPCR and Western blot analyses were performed.

\section{Results}

Compared with age-matched WKY rats, $\mathrm{SH}$ rats at 16 weeks of age exhibited significantly greater myocardial hypertrophy and remodeling with abnormal heart function. There was a reduction in autophagy and increase in apoptosis, resulting in an imbalance of oxidative stress manifested as left ventricular hypertrophy and impaired cardiac function. These effects may be related to a decrease in PTEN expression, which leads to activation of the PI3K/AKT/mTOR signaling pathway, resulting in abnormal expression of autophagy- and apoptosis-related proteins. After 12 weeks of HDR administration, blood pressure and ventricular hypertrophy were reduced, MDA and SOD levels and NADPH oxidase activity were better regulated, and gene expression of myocardial hypertrophy markers (ANP and $\beta-M H C$ ) was inhibited. HDR can promote autophagy and inhibit apoptosis, which may be related to regulation of autophagy- and apoptosis-related genes and proteins. HDR can also induce autophagy by enhancing expression of PTEN and inhibiting activation of the PI3K/AKT/mTOR signal pathway. Importantly, we demonstrated that VO-Ohpic, an inhibitor of PTEN, could suppress the effect of HDR on LVH in spontaneously hypertensive rats.

\section{Conclusion}


In conclusion, these results provide evidence for an important role of HDR in inhibition of left ventricular hypertrophy in spontaneously hypertensive rats, and indicate that it may act by improving autophagy through the PTEN/PI3K/AKT /mTOR pathway.

\section{Introduction}

Epidemiological studies have demonstrated that left ventricular hypertrophy (LVH) develops in $>35 \%$ of hypertensive individuals and is a key risk factor for heart failure. Previous data have shown that reduction in LV mass lessens cardiovascular complications [1]. To more effectively prevent cardiac hypertrophy and more successfully apply therapeutic interventions, it is important to better understand factors involved in ventricular growth at an early stage of cardiac hypertrophy, rather than after it is established, so that therapeutic interventions can be more successfully applied [2]. However, the mechanism of hypertensioninduced LVH remains unclear.

In a recent study, we observed that profound changes in autophagy are present in the early stages of hypertension, before manifestation of LVH and cardiac dysfunction, in hearts of spontaneously hypertensive $(\mathrm{SH})$ rats [3]. This sequence of events supports the concept of impaired autophagy preceding hypertension-induced LVH. Accordingly, targeting autophagy may offer a novel approach to preventing cardiac contractile dysfunction and structural remodeling in patients with hypertension [4]. Recent studies have shown that exercise-induced cardioprotection results from upregulation of autophagy in both acute and endurance exercise $[5,6]$. Over the last decade, much research has focused on identifying the signaling pathways that regulate cardiac hypertrophy [7]. Among these pathways, the mammalian target of rapamycin (mTOR) has emerged as a potentially important regulator of cardiac hypertrophy.

Besides increasing cell size, cardiac hypertrophy involves increased protein synthesis controlled by the $\mathrm{PI} 3 \mathrm{~K} / \mathrm{AKT} / \mathrm{mTOR}$ pathway [8]. AKT is a key regulator of cell growth and generally works via several downstream effectors such as mTOR, GSK-3 $\beta$, and FOXO proteins, ultimately resulting in cardiac hypertrophy [9]. Phosphatase and tensin homolog on chromosome ten (PTEN) is a tumor suppressor protein and is considered to be a vital regulator of cell viability and apoptosis. PTEN also is a negative regulator of AKT signaling and reportedly suppresses cardiac hypertrophy [10]. PTEN inhibition in mice promotes cardiac hypertrophy and a marked decrease in cardiac contractility [11]. Interestingly, other studies [12] have revealed that traditional Chinese medicine (TCM) can enhance the expression of PTEN in myocardial, vascular, brain and kidney tissue, but the precise mechanism requires further investigation.

In China, TCM has attracted increasing attention for its associated multi-potent properties [13]. It is well known that Chinese herbal medicine is effective in amelioration of LVH. TCM can interact with various pathways to improve left ventricular hypertrophy (LVH) during hypertension-associated pathological changes [14]. Clinically, the HuoXue DiTan method, a TCM herbal compound, could lower blood pressure, improve oxidative stress and reverse LVH among hypertensive patients [15]. However, the specific molecular mechanisms responsible for its effects have not been fully elucidated. Based on the evidence 
discussed above, the present study was designed to explore whether the HuoXue DiTan recipe (HDR) can alleviate LVH in spontaneously hypertensive rats by improving autophagy through the PTEN/PI3K/AKT/mTOR signaling pathway. Our research may provide a theoretical basis for the pharmacological effects of the HDR on vascular remodeling and left ventricular hypertrophy in patients with essential hypertension.

\section{Materials And Methods}

\section{Materials}

Animals and Treatments: The male WKY rats used in this study were purchased from the Chinese Academy of Sciences Shanghai Experimental Animal Center at 16 weeks of age with a body weight $300 \pm 20 \mathrm{~g}$ and a clean grade with certificate: SCXK (Shanghai) 2003-0003. The male SH rats used in this study were purchased from Beijing Wei Tong Lihua Experimental Animal Center at 16 weeks of age with a body weight $300 \pm 20 \mathrm{~g}$ and a clean grade with certificate: SCXK (Beijing) 2007-2001. WKY rats and SH rats were housed until 21 weeks of age in a clean environment with a light/dark cycle of $12 \mathrm{~h} / 12 \mathrm{~h}$, relative humidity of $50-60 \%$, ambient temperature $22-25^{\circ} \mathrm{C}, 4$ rats per cage, and free access to food and water. All procedures were approved by the Animal Care and Use Committee of Central South University.

The SH rats were randomized into three groups of ten each: (1) The SH group was treated with distilled water and served as an untreated control, (2) the HDR group was treated only with HDR, $6.48 \mathrm{mg} / \mathrm{kg} / \mathrm{d}$, and (3) the inhibitor group was treated with both HDR, $6.48 \mathrm{mg} / \mathrm{kg} / \mathrm{d}$, and the PTEN inhibitor VO-OHpic, $10 \mathrm{mg} / \mathrm{kg} / \mathrm{d}$, via intraperitoneal injection every two weeks from the beginning of the experiment [16]. Every group was given daily oral lavage with the appropriate designated drug for 12 weeks. The WKY rats (WKY group) were also treated with distilled water as a blank control. Rat body weight, systolic blood pressure (SBP) and diastolic blood pressure (DBP) were measured each day for two weeks, and systolic cardiac function was monitored via echocardiography twice, at the beginning and end of the experiment. All rats survived the study, and no significant abnormalities were observed.

\section{Experimental reagents}

VO-OHpic was obtained from Sigma-Aldrich (St. Louis, MO, USA). Trizol was from Invitrogen Inc., USA. Diethylpyrocarbonate (DEPC) and PCR primers were from Beijing Parkson Gene Technology Ltd, China. dNTP, RNAsin, Taq DNA polymerase, and DNA ladder were from Sino-American Inc, Beijing, China. The primary antibodies were from ThermoFisher Scientific Inc., USA. The malondialdehyde (MDA) assay kit (A003-1-2), nicotinamide adenine dinucleotide phosphate (NADPH) kit (A127-1-1), and superoxide dismutase (SOD) kit (A001-3-2) were purchased from the Nanjing Jiancheng Biology Engineering Institute. The TUNEL apoptosis detection kit (C1086) was purchased from Shanghai Beyotime Biotechnology Co., Ltd. The CFX 96 touch fluorescence quantitative PCR detection system was obtained from Bio-Rad (United States), and the LSM510 laser confocal microscope from Carl Zeiss (Germany). 
HDR consists of Salvia miltiorrhiza, $15 \mathrm{~g}$, sappanwood, $10 \mathrm{~g}$, Pinellia ternata, $10 \mathrm{~g}$, tangerine peel, $10 \mathrm{~g}$, Poria cocos, $9 \mathrm{~g}$, and licorice root, $5 \mathrm{~g}$. The Pharmacy Department of the Xiangya Hospital of Central South University purchased, mixed, decocted, filtered, concentrated and dried the six herbs and extracted the powder according to standard procedures. Finally, we obtained $3.75 \mathrm{~kg}$ powder containing $6.48 \mathrm{~g}$ of crude medicine per gram of powder. Briefly, according to the Chinese Pharmacopoeia (2015 edition), tanshinone $\triangle A$ and protosappanin $A$ were quantified for quality control. Their yield was 0.35 and 0.42 $\mathrm{mg} / \mathrm{g}$, respectively, as measured by high performance liquid chromatography (HPLC). Standard samples of tanshinone $\mathbb{A}$ and protosappanin A were purchased from Chengdu Gelipu Bio-tech Co., Ltd.(Chengdu, China).

\section{Methods}

\section{Measurement of tail artery blood pressure}

Systolic blood pressure (SBP) and diastolic blood pressure (DBP) were measured weekly in the tails of conscious rats using a noninvasive computerized tail-cuff system (Kent Scientific Corporation, CT, USA). The rats were warmed at $28^{\circ} \mathrm{C}$ for $10-15$ min before the measurements to allow for detection of tail artery pulsations and to achieve a steady pulse. To minimize stress-induced fluctuations, the rats were pre-trained by measuring blood pressure daily for at least 1 week before the experiments began. Tail artery blood pressure was averaged over five measurements.

\section{Echocardiography}

After the 12-week drug administration period, transthoracic echocardiography was performed under isoflurane anesthesia using an ultrasound system (Vevo 2100, VisualSonics, Toronto, Canada) with a 21 $\mathrm{MHz}$ probe. LV mass and LV volume during diastole (LVVd) and systole (LVVs) were measured. The LV mass-to-body weight ratio and ejection fraction (EF) were calculated [17]. Measurements were averaged over three consecutive cardiac cycles.

\section{Blood sample analysis}

At the end of the observation, blood samples were collected. Serum samples were obtained by centrifugation of the blood samples at $3000 \mathrm{rpm}$ for $10 \mathrm{~min}$ at $4^{\circ} \mathrm{C}$. The supernatant was collected and immediately stored at $-80^{\circ} \mathrm{C}$, and thawed at $-4^{\circ} \mathrm{C}$ before analysis. The serum levels of MDA and SOD, and the activity of NADPH oxidase in myocardial tissue, were determined using the appropriate biochemical kits.

\section{Measurement of left ventricular mass index}

When all measurements were complete, the chest of the rat was immediately opened and the heart quickly removed. After drying with filter paper, the remaining septal and left ventricular free walls were weighed to determine left ventricular mass. The left ventricular mass index (LVMI) was calculated as left ventricular mass/body mass $(\mathrm{mg} / \mathrm{g})$. The central section of the left ventricle was harvested and fixed in $10 \%$ neutral formalin, and the remaining portion was snap frozen in liquid nitrogen and stored at $-80^{\circ} \mathrm{C}$. 


\section{Histological analyses}

\section{(1)Hematoxylin-eosin staining}

Heart sections ( $5 \mu \mathrm{m}$ thick) were examined by hematoxylin-eosin (HE) staining (Service Biological Technology Co., Ltd, Wuhan, China) to measure the cross-sectional area of cardiomyocytes. Three to five random fields (around 30-50 cells per field) were selected from each of three sections from each animal for observation under a light microscope (Olympus Corporation, Tokyo, Japan).

\section{(2)Masson's trichrome staining}

The sections were placed in composite Masson's staining solution for $5 \mathrm{~min}$, washed in a $0.2 \%$ acetic acid solution for $1 \mathrm{~min}$, stained with $5 \%$ phosphotungstic acid for 5 min, dipped in $0.2 \%$ acetic acid solution for $2 \mathrm{~min}$, stained with brilliant green staining solution for $5 \mathrm{~min}$, dipped in $0.2 \%$ acetic acid solution twice, separated in $95 \%$ alcohol, dehydrated in gradient alcohol, cleared with xylene, and mounted with neutral gum. Masson's trichrome stained myocardial cells red and collagen green. Collagen content was observed under a light microscope, and 10 random views were analyzed using image analysis software (Image-Pro Plus, Media Cybernetics, USA).

\section{(3)Transmission electron microscopy}

Left ventricular tissue was cut into cubes of approximately $1 \mathrm{~mm}$ on a side and fixed with $2.5 \%$ glutaraldehyde in $0.1 \mathrm{~mol} / \mathrm{L}$ phosphate buffer overnight at $\mathrm{pH} 7.4$ and $4^{\circ} \mathrm{C}$. Subsequently, the sections were put into $1 \%$ osmium tetroxide for 2 hours and then dehydrated in a graded ethanol series. After embedding in epoxy resin, ultra-thin sections $(60-70 \mathrm{~nm})$ were post-stained with uranyl acetate and lead citrate. Finally, the sections were analyzed using a JEM-1010 transmission electron microscope (JEOL Ltd, Tokyo, Japan).

\section{(4)TUNEL assay}

Three paraffin sections randomly picked from left ventricular tissue were analyzed by the terminal deoxynucleotidyl transferase-mediated dUTP nick end labeling (TUNEL) assay according to the manufacturer's instructions as previously described [18]. The TUNEL index (\%) was calculated as the ratio of the number of TUNEL-positive cells divided by the total number of cells. At least 3 representative fields were evaluated from each experimental group, from which an average value was calculated.

\section{(5)Immunohistochemical staining}

The myocardial tissue samples were embedded in paraffin and sliced into $4 \mu \mathrm{m}$-thick sections. After being deparaffinized and rehydrated, sections were blocked with $5 \%$ goat serum for $1.5 \mathrm{~h}$, and incubated overnight at $4^{\circ} \mathrm{C}$ with $\beta$-myosin heavy chain ( $\beta$-MHC) monoclonal antibodies (1:300, Abcam Technology, Inc. Cambridge, MA, USA). After washing with PBS, a secondary antibody were sequentially incubated at $37^{\circ} \mathrm{C}$ for $45 \mathrm{~min}$, washed with PBS, and stained with diaminobenzidine (DAB) (K5007, DAKO, Germany). Cells in 6 random fields of each section were counted at $\times 400$ magnification. The optical density (OD) of 
positive cells was analyzed by Image-Pro Plus 6.0 software. The average integrated optical density (AIOD) was calculated as follows: AIOD = positive area $\times$ OD/total area.

\section{(6)Immunofluorescence staining}

Cryosections of the heart ( $4 \mu \mathrm{m}$ thick) were thawed and fixed in ice-cold acetone for $10 \mathrm{~min}$. Nonspecific binding was blocked in $5 \%$ BSA for $1 \mathrm{~h}$. Tissue sections were incubated with rabbit anti-PTEN antibody (1:500; Abcam Technology, Inc., Cambridge, MA, USA). After being rinsed with PBS, tissue sections were incubated with secondary antibody conjugated to FITC (fluorescein isothiocyanate) (1:100). Cell nuclei were stained with DAPI (4',6-diamidino-2-phenylindole) (1:1000) at room temperature for 10 min after washing with PBS. Replacement of the primary antibody with isotype IgG acted as a negative control. Fluorescence signals were observed with an optical microscope (BX51; Olympus, Tokyo, Japan).

\section{Quantitative real-time PCR}

Total tissue RNA was purified from hearts using Trizol (Invitrogen, Carlsbad, CA, USA), and cDNA was synthesized using the GoScript ${ }^{\mathrm{TM}}$ reverse transcription system (Promega, Southampton, UK). Quantitative real-time PCR (qPCR) was performed using SYBR Green Master Mix (Takara, Kusatsu, Japan) on an Applied Biosystems 7500 Fast System (ABI, Carlsbad, CA, USA) as described previously [19]. The genespecific primer sequences (TaKaRa Bio) are shown in Table S1. GAPDH was used as an internal control. The primers were obtained from Sangon Biotech (Shanghai, China). Relative mRNA levels were calculated using the $2^{\Delta \Delta C t}$ method as described previously [19].

\section{Western blotting}

Total protein was extracted from heart tissues using lysis buffer containing protease/phosphatase inhibitors (Thermo Fisher Scientific, Carlsbad, CA, USA). Protein concentrations were assessed using the Abbkine Protein Quantification Kit (Thermo Fisher Scientific, Carlsbad, CA, USA). Equal amounts of proteins $(40-50 \mu \mathrm{g}$ ) were separated by SDS-PAGE (sodium dodecyl sulfate-polyacrylamide gel electrophoresis) and transferred onto a PVDF (polyvinylidene fluoride) membrane (Millipore, Billerica, MA, USA). Immunoblotting analysis was performed as described previously [20]. All protein levels were normalized to GAPDH. Images were captured and quantified by FluorChem M (ProteinSimple, San Jose, CA, USA). The primary antibodies are listed in Table S2. After washing with TBST (tris buffered saline with tween), secondary antibody (1:200) was added, and the blots were incubated at room temperature for $3 \mathrm{~h}$ before again being washed with TBST. ECL (enhanced chemiluminescence) developing solution was added to the membranes, which were then exposed to X-ray film for 5-8 min. Quantity One analysis software (Bio-Rad, Hercules, CA) was used to analyze the results, and the integrated optical density (IOD) value was obtained by dividing the level of the target protein by that of the internal reference band (GAPDH) and used as the final result for statistical analysis.

\section{Statistical analyses}

Data are presented as mean \pm standard error of the mean (SEM). Using GraphPad Prism 4.0 (GraphPad software Inc., CA, USA), statistical significance among multiple groups was evaluated by one-way 
analysis of variance (ANOVA) with the Bonferroni post hoc test. A two-tailed P value $<0.05$ was considered statistically significant.

\section{Results}

\section{(1) Administration of HDR attenuated hypertension and cardiac dysfunction in SH rats}

As shown in Fig. 1A, SBP and DBP increased significantly in the SH group compared with the WKY group throughout the experiment $(P<0.01)$; Compared with the $\mathrm{SH}$ group, the HDR group had lower SBP and DBP over a period of $4-12$ weeks $(P<0.05)$. However, when the PTEN inhibitor was added to the TCM intervention (HDR-treated) group, systolic and diastolic blood pressure increased significantly over the same period $(P<0.05)$. Echocardiographic parameters for these treatments are shown in Figure 1B-D. Significant differences were observed between WKY and $\mathrm{SH}$ rats for left ventricular posterior wall thickness during diastole (LVPWd) $(P<0.01)$, interventricular septal thickness during diastole (IVSd) $(\mathrm{P}<$ 0.01), left ventricle end-diastolic dimension (LVDd) $(P<0.01)$, left ventricle volume (LVD.vol) $(P<0.01)$, and left ventricle ejection fraction (LVEF) $(P<0.01)$. HDR treatment resulted in a significant decrease in these differences (LVPWd, $\mathrm{P}<0.05$; IVSd, $\mathrm{P}<0.05$; LVDd, $\mathrm{P}<0.01$; LVD.vol, $\mathrm{P}<0.05$; and LVEF, $\mathrm{P}<0.05$ ). However, when the PTEN inhibitor was added to treatment of the TCM intervention group, there was a significant decrease in these differences between the HDR-treated and inhibitor groups (LVPWd, $P<0.05$; IVSd, $\mathrm{P}<0.05$; LVDd, $\mathrm{P}<0.01$; LVD.vol, $\mathrm{P}<0.05$; and LVEF, $\mathrm{P}<0.05$ ). To further confirm the role of HDR in $\mathrm{SH}$ rats with ventricular hypertrophy, mRNA expression of the hypertrophic markers $\beta-\mathrm{MHC}$ and ANP was detected by qRT-PCR, and $\beta-M H C$ protein expression was assessed by immunohistochemical analysis (Fig. 2). Expression of ANP and $\beta$-MHC significantly increased in the SH group compared with the WKY group $(P<0.01)$, while they significantly decreased in the HDR group compared with the $\mathrm{SH}$ group $(P<0.01)$, and this was largely reversed by the PTEN inhibitor for both ANP $(P<0.05)$ and $\beta$-MHC $(P<0.01)$. These results suggest that HDR markedly improved blood pressure and cardiac function in $\mathrm{SH}$ rats, and that the observed effects may be associated with the PTEN signaling pathway

\section{(2) Administration of HDR alleviated left ventricular hypertrophy and fibrosis in $\mathrm{SH}$ rats}

Coronary sections of the heart are shown in Fig. 3A-B. Compared with WKY rats, SH rats had significantly higher heart size $(P<0.05)$, heart weight $(H W)$ /body weight $(B W)$ ratio $(P<0.01)$, and $H W /$ tibial length $(T L)$ ratio $(P<0.01)$. The ratio of $H W / B W$ and $H W / T L$ significantly decreased in the HDR group compared with the $\mathrm{SH}$ group $(P<0.01, P<0.05)$, which was largely reversed by treatment with the PTEN inhibitor $(P<0.05)$. Histopathological analysis using HE (Fig. 3C) and Masson's trichrome (Fig. 3D) staining revealed that cardiomyocyte area and collagen deposition were higher in $\mathrm{SH}$ rats than in WKY rats $(\mathrm{P}<0.01)$ (Fig. $3 \mathrm{E}-$ F). However, the two quantities significantly decreased in the HDR group compared with the SH group $(P<0.01, P<0.05)$, which was largely reversed by the PTEN inhibitor $(P<0.01)$. These results show that HDR 
markedly reduced ventricular hypertrophy and fibrosis in SH rats, and that the observed effects may be associated with the PTEN signaling pathway.

\section{(3) HDR attenuates oxidative stress in SH rats with ventricular hypertrophy.}

ELISA was used to detect the level of SOD and MDA in serum, and the activity of NADPH oxidase in heart tissue. The activity of NADPH oxidase was significantly greater in the SH group than in the WKY group $(P<0.01$; Fig. 4D). The levels of SOD and MDA were significantly less in the SH group than in the WKY group $(P<0.01)$. Following HDR treatment, the activity of NADPH oxidase significantly decreased, and SOD and MDA levels significantly increased, over those observed in the $S H$ group $(P<0.05, P<0.01)$. Following treatment with PTEN inhibitor, the effect of HDR was reduced. These results suggested that following treatment with HDR, cardiomyocyte antioxidative activity increased and oxidative stress injury in ventricular hypertrophy was attenuated.

\section{(4) HDR increased autophagy and prevented apoptosis in $\mathrm{SH}$ rats with ventricular hypertrophy.}

An increase in apoptosis in pathological cardiac hypertrophy was observed in $\mathrm{SH}$ rats in the present study. Results of the TUNEL assay (Fig. 4A-B) showed that in comparison with WKY rats, the percentage of TUNEL-positive cells was higher in SH rats, which was accompanied by an increase in expression of the apoptosis-related proteins Bcl-2, p53 and caspase-12. Treatment with HDR for 12 weeks reversed the increase in the Bcl-2, p53 and caspase-12 expression in SH rats and reduced the percentage of TUNELpositive cells. Following treatment with the PTEN inhibitor, the effect of HDR was reduced. These results indicate that HDR attenuates cardiomyocyte apoptosis in $\mathrm{SH}$ rats with ventricular hypertrophy. Next, we wanted to confirm that autophagy was in fact upregulated after HDR treatment in ventricular hypertrophy (Figure 4E). Expression of autophagy-related proteins was assayed (Figure 4F-G). SH rats exhibited a significant downregulation of Beclin 1 and LC3区/LC3\ expression $(P<0.05)$ and upregulation of p62 expression $(P<0.01)$ compared with WKY group. However, the HDR-treated groups showed upregulation of Beclin 1 and LC3区/LC3囚 expression $(P<0.05)$ and downregulation of p62 expression $(P<0.01)$ compared with the SH group. Following treatment with the PTEN inhibitor, the effect of HDR was reduced. These results suggest that HDR markedly increased autophagy and prevented apoptosis in SH rats with ventricular hypertrophy, and that the observed effects may be associated with the PTEN signaling pathway.

\section{(5) HDR increased autophagy through the $\mathrm{PTEN} / \mathrm{PI}$ 3K/AKT/mTOR pathway in SH rats with ventricular hypertrophy.}


To identify the mechanism by which HDR increased autophagy in SH rats with ventricular hypertrophy, expression of PTEN mRNA and protein was detected (Fig. 5A-C). PTEN expression significantly decreased in the SH group compared with the WKY group $(P<0.01)$. In addition, the HDR-treated group exhibited upregulation of PTEN expression $(P<0$.05) compared with the $\mathrm{SH}$ group. Following treatment with the PTEN inhibitor, the effect of HDR was reduced. Furthermore, we measured expression of $\mathrm{PI} 3 \mathrm{~K} / \mathrm{AKT} / \mathrm{mTOR}$ signaling pathway-associated proteins, including PI3K, AKT and mTOR, in rat myocardial tissue (Fig. 5E-F). Expression of the phosphorylated proteins, p-PI3K, p-AKT and p-mTOR, significantly increased in the SH group compared with the WKY group $(P<0.05, P<0.01$, respectively). In addition, the HDR-treated group exhibited downregulation of p-PI3K, p-Akt and pmTOR expression $(\mathrm{P}<0$ .05) compared with the SH group. Following treatment with the PTEN inhibitor, the effect of HDR was reduced. These data suggest that the PTEN/PI3K/AKT/mTOR signaling pathway plays a key role in autophagy in $\mathrm{SH}$ rats with ventricular hypertrophy.

\section{Discussion}

The exact mechanism by which hypertension results in ventricular hypertrophy remains unclear. The molecular mechanisms of ventricular hypertrophy involve multiple factors, including accumulation of reactive oxygen species, induced apoptosis, DNA damage, and mitochondrial dysfunction [21]. Autophagy dysfunction plays an important role in ventricular hypertrophy resulting from essential hypertension [22]. The aim of the present study was to elucidate the impacts of the HDR on ventricular hypertrophy in $\mathrm{SH}$ rats by examining autophagy. Furthermore, we examined the potential cardioprotective effect of the HDR via inhibition of the PTEN/PI3K/AKT/mTOR signaling pathway, increased autophagy and prevention of oxidative stress in SH rats. Our data demonstrated that oral administration of the HDR could attenuate pressure overload-induced ventricular hypertrophy via inhibition of the PTEN/PI3K/AKT/mTOR signaling pathway.

Cardiac remodeling is one of the most common complications associated with hypertension, and it is likely to be a risk factor that is independent of blood pressure [23]. At the age of 16 weeks, in comparison with age-matched WKY rats, $\mathrm{SH}$ rats exhibited significant myocardial hypertrophy and remodeling with abnormal heart function. Previous studies have shown that oxidative stress plays an important role in left ventricular hypertrophy with hypertension [24]. MDA and SOD are markers of oxidative stress, and NADPH oxidase is related to reactive oxidase stress in activated RAAS $[25,26]$. In the present study, the ELISA assay revealed that the activity of NADPH oxidase significantly increased, the levels of SOD and MDA significantly decreased, and expression of ANP and $\beta-\mathrm{MHC}$, indicators of pathological cardiac hypertrophy, increased in cardiomyocytes in SH rats as compared with WYK rats. HDR administration to $\mathrm{SH}$ rats reduced blood pressure, improved EF, reduced heart weight and cardiomyocyte cross-sectional area, inhibited oxidative stress, and downregulated gene expression of myocardial hypertrophy markers (ANP and $\beta-\mathrm{MHC}$ ). These results strongly suggest that HDR treatment improved heart function and reduced cardiac hypertrophy associated with hypertension. 
HDR is derived from a classic prescription of TCM. Previous studies have shown that TCM (Salvia miltiorrhiza, hematoxylin, Pinellia ternata and Poria cocos) can significantly improve left ventricular hypertrophy caused by hypertension through a variety of genes and signaling pathways [27-30]. To explore the mechanism by which HDR improves ventricular hypertrophy caused by hypertension, we studied genes and signaling pathways related to cardiomyocyte apoptosis and autophagy in ventricular hypertrophy. In addition, the TUNEL assay revealed that the hearts of $\mathrm{SH}$ rats contained greater numbers of apoptotic cells. To determine the molecular mechanism of these changes, we examined gene markers for apoptosis, anti-apoptosis, and cell survival. In SH rats, expression of the genes for the proapoptotic markers p53 and Bcl 2 was upregulated. Interestingly, we found that treatment of SH rats with HDR resulted in a significant reduction in expression of these proapoptotic markers, leading to increased cardiomyocyte survival, consistent with previous observations [31]. Caspase 12 expression plays an important role in mitochondria-dependent apoptosis [32]. In this study, SH rats exhibited upregulated caspase 12 protein expression. In contrast, the HDR-treated SH group exhibited reduced caspase 12 protein expression. These data demonstrated that HDR could attenuate cardiac apoptosis by inhibiting expression of survival proteins.

Autophagy is a highly conserved and ubiquitous metabolic pathway in living organisms [33]. Previous studies have demonstrated that Beclin 1 is the earliest self-localizing gene in the structure of autophagic precursors and is believed to regulate other autophagic genes, cardiac-specific overexpression of Beclin-1 promoted autophagy, and improved cardiac function [34, 35]. We observed a dramatic decrease in LC3II and Beclin 1 in LVH of SH rats. In addition, 12 weeks of HDR treatment induced activation of the autophagic pathway in $\mathrm{SH}$ rat ventricles, including enhanced expression of Beclin 1 and an increased LC3-II/LC3-I ratio. Knock-out or silencing of the p62 gene in cardiomyocytes decreased autophagic activity [36]. We then measured expression of p62 and found that its gene was also obviously upregulated in LVH. In contrast, we found that the expression of p62 was significantly downregulated at the protein level in the left ventricles of rats with HDR-induced myocardial hypertrophy. These findings are consistent with previous results demonstrating that autophagy is enhanced by TCM [37], and that Salvia miltiorrhiz provides cardioprotection by upregulating autophagy [38]. Taken together, these results suggest that HDR can increase autophagy and attenuate apoptosis in cardiomyocytes, which play an important role in pressure-overload cardiac hypertrophy.

Regulation of autophagy is rather complicated. The key players in initiation of autophagy are mTORrelated targets [39] regulated by signaling pathways such as the PI3K/AKT pathway [40], which is the main regulator of cell growth and survival [41] and plays an important role in survival of cardiomyocytes. Activation of the PI3K/Akt pathway phosphorylates mTOR, thereby inhibiting autophagy [42]. The $\mathrm{PI3K} /$ Akt pathway is one of the most important upstream pathways of mTOR [43]. Poria cocos, Glycyrrhiza uralensis and Salvia miltiorrhiza, the main ingredients of HDR, can upregulate autophagy through the PI3K/Akt pathway signal [44-46].

Given the above relationships, we focused our attention on mTOR, the major regulator of autophagy in cells. TCM treatment of different cell types has shown a clear decrease in the level of p-mTOR [47, 48]. 
Thus, mTOR may be a direct target of TCM, similar to the effect of rapamycin. However, upstream of mTOR, a sharp decrease in the expression of both active forms of AKT, p-AKT (S473) and p-AKT (T308), is observed in response to TCM [49]. These observations led us to believe that downregulation of this pathway may be targeted through PTEN, a natural inhibitor of the PI3K-AKT pathway [50]. PTEN is a tumor suppressor gene that can induce autophagy through the PI3K-AKT pathway [10]; in contrast, its suppression can inhibit autophagy [51]. Our data also suggested a major role for PTEN in HDR-induced autophagy in SH rats, wherein HDR treatment led to greatly enhanced expression of PTEN in myocardial tissue, resulting in suppression of the AKT pathway and induction of autophagy. This finding is consistent with our observation that HDR exhibits a similar mechanism related to induction of autophagy in different cell types [52]. To confirm the involvement of PTEN in HDR-induced autophagy, we attempted to knock down expression of PTEN with an inhibitor (VO-OHpic). Interestingly, HDR could increase cell autophagy in $\mathrm{SH}$ rats, as indicated by a sharp increase in autophagic flux. However, HDR did not elicit the autophagic response in the PTEN inhibitor group, consistent with the observations above [53]. Thus, we conclude that PTEN-mediated inhibition of the PI3K/AKT/mTOR pathway plays a role in induction of autophagy by HDR. We only investigated the effects of HDR on signaling pathways, but not its influence on blood vessels, which are important sites of changes leading to cardiac remodeling. We expect that additional mechanisms of HDR treatment will be investigated in future experiments.

\section{Abbreviations}

LVH Left ventricular hypertrophy

PTEN Phosphatase and tensin homolog on chromosome ten

PI3K Phosphatidylinositol trihydroxykinase

AKT Protein Kinase B

mTOR mammalian target of rapamycin

HDR HuoXue DiTan recipe

SH Spontaneously hypertensive

WKY Wistar-Kyoto

MDA Malondialdehyde

SOD Superoxide dismutase

NADPH Nicotinamide adenine dinucleotide phosphate

ANP Atrial natriuretic peptide 
$\beta-\mathrm{MHC} \beta$-myosin heavy chain

TCM Traditional Chinese medicine

SBP Systolic blood pressure

DBP Diastolic blood pressure

LVVd Left ventricular volume during diastole

EF Ejection fraction

HE Hematoxylin-eosin

ELISA Enzyme linked immunosorbent assay

TUNEL Terminal deoxynucleotidyl transferase-mediated dUTP-biotin nick end labeling assay

RAAS Renin-angiotensin-aldosterone system

\section{Conclusions}

In conclusion, the present investigation suggests that HDR alleviates cardiac remodeling in pressureoverloaded rat ventricles. HDR attenuated cardiac hypertrophy, interstitial fibrosis, myocyte apoptosis and oxidative stress, and subsequently increased autophagy and improved LV function. The cardioprotective effect of HDR is associated with inhibition of the PTEN/PI3K/AKT/mTOR signaling pathways.

\section{Declarations}

\section{Ethics approval and consent to participate}

Animal Care and Use Committee of Central South University and conducted in accordance with the Guide for the Care and Use of Laboratory Animals. The ethical approval number is 20181115012 and was approved in November 2018.

\section{Consent to publish}

Not applicable.

\section{Availability of data and materials}

The datasets used and/or analyzed during the current study are available from the corresponding author on reasonable request.

Competing interests 
The authors have declared that no competing interest exists.

\section{Funding}

This work was financially supported by Natural Science Foundation of China(Grant Nos.

81673858,81704062 ) and the project of Natural Science Foundation of Hunan Province and Scientific Research Project of Hunan Provincial Health Commission(Grant Nos. 2021JJ31118, 2020JJ8073) and the Program for National Center for Clinical Medicine for Geriatric Diseases (Ministry of Science and Technology).

\section{Authors' Contributions}

G.Z. conceived and designed the entire study, writing-review \& editing. J.Y. analyzed the data and performed literature research, writing-review \& editing. C.Z. and Y.Y. performed literature research and drafted the paper. H.Y., Y. S and D. Z. Supervision, Validation, writing. G.Z. and J.Y. guided the entire study and revised it critically for important intellectual content. All authors have read and agreed with the final version of this manuscript.

\section{Acknowledgements}

Not applicable.

\section{References}

1. Soliman EZ, Prineas RJ. Antihypertensive Therapies and Left Ventricular Hypertrophy. Curr Hypertens Rep. 2017 Sep 19,19(10):79.

2. Nakamura M, Sadoshima J. Mechanisms of physiological and pathological cardiac hypertrophy. Nat Rev Cardiol. 2018 Jul,15(7):387-407.

3. Huang J, Huang H, Pan W, Ou D, Dai W, Lin Y, Wu J, Xie W, Chen X. Renal denervation attenuates cardiac hypertrophy in spontaneously hypertensive rats via regulation of autophagy. Mol Med Rep. 2017 Aug, 16(2):2023-2029.

4. Li Z, Wang J, Yang X. Functions of autophagy in pathological cardiac hypertrophy. Int J Biol Sci. 2015,11:672-678.

5. Li J, Cai Y. The dual effects of autophagy in myocardial hypertrophy. Acta Cardiol. 2015, 70:493498.

6. Lee Y, Kwon I, Jang Y, Song W, Cosio-Lima LM, Roltsch MH. Potential signaling pathways of acute endurance exercise-induced cardiac autophagy and mitophagy and its possible role in cardioprotection. J Physiol Sci. 2017 Nov,67(6):639-654.

7. Schüttler D, Clauss S, Weckbach LT, Brunner S. Molecular Mechanisms of Cardiac Remodeling and Regeneration in Physical Exercise. Cells. 2019 Sep 23,8(10):1128. 
8. Zhao LG, Li PL, Dai Y, Deng JL, Shan MY, Chen B, Zhang KB, Guo SD, Xu ZH. Mibefradil Alleviates High-Glucose-induced Cardiac Hypertrophy by Inhibiting PI3K/Akt/mTOR-mediated Autophagy. J Cardiovasc Pharmacol. 2020 Aug,76(2):246- 254.

9. Pei H, Wang W, Zhao D, Su H, Su G, Zhao Z. G Protein-Coupled Estrogen Receptor 1 Inhibits Angiotensin II-Induced Cardiomyocyte Hypertrophy via the Regulation of PI3K-Akt-mTOR Signalling and Autophagy. Int J Biol Sci. 2019 Jan 6,15(1):81-92.

10. Tian M, Jiang X, Li X, Yang J, Zhang C, Zhang W. LKB1IP promotes pathological cardiac hypertrophy by targeting PTEN/Akt signalling pathway. J Cell Mol Med. 2021 Mar,25(5):2517-2529.

11. Chen C, Zou LX, Lin QY, Yan X, Bi HL, Xie X, Wang S, Wang QS, Zhang YL, Li HH. Resveratrol as a new inhibitor of immunoproteasome prevents PTEN degradation and attenuates cardiac hypertrophy after pressure overload. Redox Biol. 2019 Jan,20:390 -401.

12. Chen $\mathrm{C}$, Zhang $\mathrm{H}, \mathrm{Xu} \mathrm{H}$, Xue R, Zheng $\mathrm{Y}$, Wu T, Lian $\mathrm{Y}$. Harpagoside Rescues the Memory Impairments in Chronic Cerebral Hypoperfusion Rats by Inhibiting PTEN Activity. J Alzheimers Dis. 2018,63(2):445-455.

13. Hao P, Jiang F, Cheng J, Ma L, Zhang Y, Zhao Y. Traditional Chinese Medicine for Cardiovascular Disease: Evidence and Potential Mechanisms. J Am Coll Cardiol. 2017 Jun 20,69(24):2952-2966.

14. Cheng Y, Shen A, Wu X, Shen Z, Chen X, Li J, Liu L, Lin X, Wu M, Chen Y, Chu J, Peng J. Qingda granule attenuates angiotensin II-induced cardiac hypertrophy and apoptosis and modulates the PI3K/AKT pathway. Biomed Pharmacother. 2021 Jan,133:111022.

15. Wang J, Dong ZH, Gui MT, Yao L, Li JH, Zhou XJ, Fu DY. HuoXue QianYang QuTan Recipe attenuates left ventricular hypertrophy in obese hypertensive rats by improving mitochondrial function through SIRT1/PGC-1a deacetylation pathway. Biosci Rep. 2019 Dec 20,39(12):BSR20192909.

16. Chen C, Zou LX, Lin QY, Yan X, Bi HL, Xie X, Wang S, Wang QS, Zhang YL, Li HH. Resveratrol as a new inhibitor of immunoproteasome prevents PTEN degradation and attenuates cardiac hypertrophy after pressure overload. Redox Biol. 2019 Jan,20:390- 401.

17. Chen Y, Chang Y, Zhang N, Guo X, Sun G, Sun Y. Atorvastatin Attenuates Myocardial Hypertrophy in Spontaneously Hypertensive Rats via the C/EBPB/PGC-1a/UCP3 Pathway. Cell Physiol Biochem. 2018,46(3):1009- 1018.

18. Luo J, Yan D, Li S, Liu S, Zeng F, Cheung CW, Liu H, Irwin MG, Huang H, Xia Z. Allopurinol reduces oxidative stress and activates Nrf2/p62 to attenuate diabetic cardiomyopathy in rats. J Cell Mol Med. 2020 Jan,24(2):1760-1773.

19. Reja V, Goodchild AK, Phillips JK, Pilowsky PM. Tyrosine hydroxylase gene expression in ventrolateral medulla oblongata of WKY and SHR: a quantitative real-time polymerase chain reaction study. Auton Neurosci. 2002 Jun 28,98(1-2):79-84.

20. Li Y, Yang X, Sun Q, Duan B, Wang Y. The bioflavonoid troxerutin prevents gestational hypertension in mice by inhibiting STAT3 signaling. Hypertens Res. 2021 Apr,44(4):399-406.

21. Schirone L, Forte M, Palmerio S, Yee D, Nocella C, Angelini F, Pagano F, Schiavon S, Bordin A, Carrizzo A, Vecchione C, Valenti V, Chimenti I, De Falco E, Sciarretta S, Frati G. A Review of the Molecular 
Mechanisms Underlying the Development and Progression of Cardiac Remodeling. Oxid Med Cell Longev. 2017,2017:3920195.

22. Li L, Xu J, He L, Peng L, Zhong Q, Chen L, Jiang Z. The role of autophagy in cardiac hypertrophy. Acta Biochim Biophys Sin (Shanghai). 2016 Jun,48(6):491-500.

23. González A, Ravassa S, López B, Moreno MU, Beaumont J, San José G, Querejeta R, Bayés-Genís A, Díez J. Myocardial Remodeling in Hypertension. Hypertension. 2018 Sep,72(3):549-558.

24. Sulistyowati E, Hsu JH, Cheng YB, Chang FR, Chen YF, Yeh JL. Indonesian herbal medicine prevents hypertension-induced left ventricular hypertrophy by diminishing NADPH oxidase-dependent oxidative stress. Oncotarget. 2017 Sep 30,8(49):86784- 86798.

25. Amin MM, Rafiei N, Poursafa P, Ebrahimpour K, Mozafarian N, Shoshtari-Yeganeh B, Hashemi M, Kelishadi R. Association of benzene exposure with insulin resistance, SOD, and MDA as markers of oxidative stress in children and adolescents. Environ Sci Pollut Res Int. 2018 Dec,25(34):3404634052.

26. Waghela BN, Vaidya FU, Agrawal Y, Santra MK, Mishra V, Pathak C. Molecular insights of NADPH oxidases and its pathological consequences. Cell Biochem Funct. 2021 Mar,39(2):218-234.

27. Zhang J, Zhang Q, Liu G, Zhang N. Therapeutic potentials and mechanisms of the Chinese traditional medicine Danshensu. Eur J Pharmacol. 2019 Dec 1,864:172710.

28. Li Yongsheng, Yan Li, Yong Yongquan. Effect of Tanshinone $\triangle A$ on the Transforming Growth Factor Beta1/Smads Signal Pathway in Rats with Hypertensive Myocardial Hypertrophy. CHINESE JOURNAL OF INTEGRATED TRADITIONAL AND WESTERN MEDICINE, 2010,30(5):499-503.

29. Chang Xinfeng, Song Chunhua, Long Xiaoying, Han Limin. Effects of Huatan Huoxue Yiqi Recipe on neuroendocrine factors and Akt signal phosphorylation in rats with myocardial hypertrophy induced by isoproterenol. Jiangxi Journal of Traditional Chinese Medicine, 2020,51(1):62-65.

30. Guo M, Liu Y, Shi D. Cardiovascular Actions and Therapeutic Potential of Tetramethylpyrazine (Active Component Isolated from Rhizoma Chuanxiong): Roles and Mechanisms. Biomed Res Int. 2016,2016:2430329.

31. Chen Ruqin, Xiong Ping, Zhou Xinxin, Liang Jiaoqiu, Wu liangzhi. Effect of Therapy of Dispelling Phlegm and Resolving Blood Stasis on Cardiac Muscle Cell Apoptosis in Hypertension Rats with Left Ventricular Hypertrophy. JOURNAL OF GUANGZHOU UNIVERSITY OF TRADITIONAL CHINESE MEDICINE, 2008,25(6):540-543.

32. Morishima N, Nakanishi K, Takenouchi H, Shibata T, Yasuhiko Y. An endoplasmic reticulum stressspecific caspase cascade in apoptosis. Cytochrome c-independent activation of caspase-9 by caspase-12.J Biol Chem. 2002 Sep 13, 277(37):34287-94.

33. Galluzzi L, Green DR. Autophagy-Independent Functions of the Autophagy Machinery. Cell. 2019 Jun 13,177(7):1682-1699.

34. Qiang Z, Jin B, Peng Y, Zhang Y, Wang J, Chen C, Wang X, Liu F. miR-762 modulates thyroxineinduced cardiomyocyte hypertrophy by inhibiting Beclin-1. Endocrine. 2019 Dec,66(3):585-595. 
35. Maejima Y, Isobe M, Sadoshima J. Regulation of autophagy by Beclin 1 in the heart. J Mol Cell Cardiol. 2016 Jun,95:19-25.

36. heng Q, Su H, Ranek MJ, Wang X. Autophagy and p62 in cardiac proteinopathy. Circ Res. 2011 Jul 22,109(3):296-308.

37. TANG Danli, LIU Zhaihua, CUI Haifen, ZHOU Yanhua, SUI Yu, ZHANG Huamin. Effects of Gualou Xiebai Banxia Decoction on the Myocardial Autophagy and Apoptosis in Rats with Myocardial Ischemia Reperfusion Injury. Journal of Basic Chinese Medicine, 2016,22(2): 177-180.

38. Wang X, Jiang Y, Zhang Q, Tian X, Sun Q, Guo D, Wang Q, Chai X, Peng L, Li C, Wang Y. Autophagy as a novel insight into mechanism of Danqi pill against post-acute myocardial infarction heart failure. $J$ Ethnopharmacol. 2021 Feb 10,266:113404.

39. Zhang G, Wang Z, Du Z, Zhang H. mTOR Regulates Phase Separation of PGL Granules to Modulate Their Autophagic Degradation. Cell. 2018 Sep 6,174(6):1492- 1506.e22.

40. Zhang Y, Kwok-Shing Ng P, Kucherlapati M, et al. A Pan-Cancer Proteogenomic Atlas of PI3K/AKT/mTOR Pathway Alterations. Cancer Cell. 2017,31(6):820-832.e3.

41. Li H, Wang R. Blocking SIRT1 inhibits cell proliferation and promotes aging through the PI3K/AKT pathway. Life Sci. 2017 Dec 1,190:84-90.

42. Liang Q, Huang X, Zeng C, Li D, Shi Y, Zhao G, Zhong M. BW373U86 upregulates autophagy by inhibiting the PI3K/Akt pathway and regulating the mTOR pathway to protect cardiomyocytes from hypoxia-reoxygenation injury. Can J Physiol Pharmacol. 2020 Oct,98(10):684-690.

43. Liu Min, Zhu Hongqiu, Li Yin, Zhu Ying, Hu Xiaodan.. Effect of Guizhi Fulingwan on Ovulation Dysfunction in PCOS-IR Rats by Regulating PI3K/Akt/mTOR Pathway. Chinese Journal of Experimental Traditional Medical Formulae. 2021, 27(6):7-14.

44. Xu H, Wang Y, Jurutka PW, Wu S, Chen Y, Cao C, Chen G, Tian B, Wang S, Cheng S. 16aHydroxytrametenolic Acid from Poria cocos Improves Intestinal Barrier Function Through the Glucocorticoid Receptor-Mediated PI3K/Akt/NF-kB Pathway. J Agric Food Chem. 2019 Oct 2,67(39):10871-10879.

45. Hwang CK, Chun HS. Isoliquiritigenin isolated from licorice Glycyrrhiza uralensis prevents 6hydroxydopamine-induced apoptosis in dopaminergic neurons. Biosci Biotechnol Biochem. 2012,76(3):536-43.

46. Cui G, Shan L, Hung M, Lei S, Choi I, Zhang Z, Yu P, Hoi P, Wang Y, Lee SM. A novel Danshensu derivative confers cardioprotection via PI3K/Akt and Nrf2 pathways. Int J Cardiol. 2013 Sep 30,168(2):1349-59.

47. Fan C, Tang X, Ye M, Zhu G, Dai Y, Yao Z, Yao X. Qi-Li-Qiang-Xin Alleviates Isoproterenol-Induced Myocardial Injury by Inhibiting Excessive Autophagy via Activating AKT/mTOR Pathway. Front Pharmacol. 2019 Nov 12,10:1329.

48. Chen ST, Lee TY, Tsai TH, Huang YC, Lin YC, Lin CP, Shieh HR, Hsu ML, Chi CW, Lee MC, Chang HH, Chen YJ. Traditional Chinese medicine Danggui Buxue Tang inhibits colorectal cancer growth through induction of autophagic cell death. Oncotarget. 2017 Aug 3,8(51):88563-88574. 
49. Meric-Bernstam F, Akcakanat A, Chen H, Do KA, Sangai T, Adkins F, Gonzalez-Angulo AM, Rashid A, Crosby K, Dong M, Phan AT, Wolff RA, Gupta S, Mills GB, Yao J. PIK3CA/PTEN mutations and Akt activation as markers of sensitivity to allosteric mTOR inhibitors. Clin Cancer Res. $2012 \mathrm{Mar}$ 15,18(6):1777-89.

50. Ye YT, Zhong W, Sun P, Wang D, Wang C, Hu LM, Qian JQ. Apoptosis induced by the methanol extract of Salvia miltiorrhiza Bunge in non-small cell lung cancer through PTEN-mediated inhibition of PI3K/Akt pathway. J Ethnopharmacol. 2017 Mar 22,200:107-116.

51. Wani A, Gupta M, Ahmad M, Shah AM, Ahsan AU, Qazi PH, Malik F, Singh G, Sharma PR, Kaddoumi A, Bharate SB, Vishwakarma RA, Kumar A. Alborixin clears amyloid- $\beta$ by inducing autophagy through PTEN-mediated inhibition of the AKT pathway. Autophagy. 2019 Oct,15(10):1810-1828.

52. Li Hong, Liu Yeqian, Gong Shan, Liu Lin, Zeng Yong, Cai Xiao, et al. Mechanism of Compound Qishao Jiangya tablets in the treatment of spontaneously hypertensive rats based on AKT/mTOR signal pathway. Journal of Beijing University of Traditional Chinese Medicine, 2020,44(4):296-303.

53. Jin L, Zhou Y, Han L, Piao J. MicroRNA302-367-PI3K-PTEN-AKT-mTORC1 pathway promotes the development of cardiac hypertrophy through controlling autophagy. In Vitro Cell Dev Biol Anim. 2020 Feb,56(2):112-119.

\section{Figures}


A
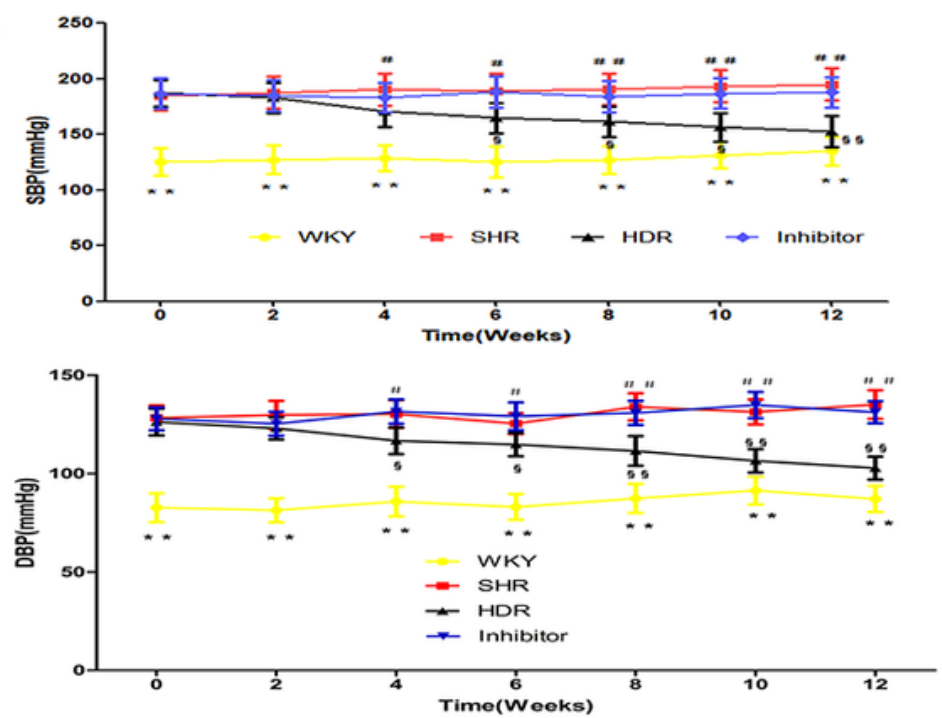

B

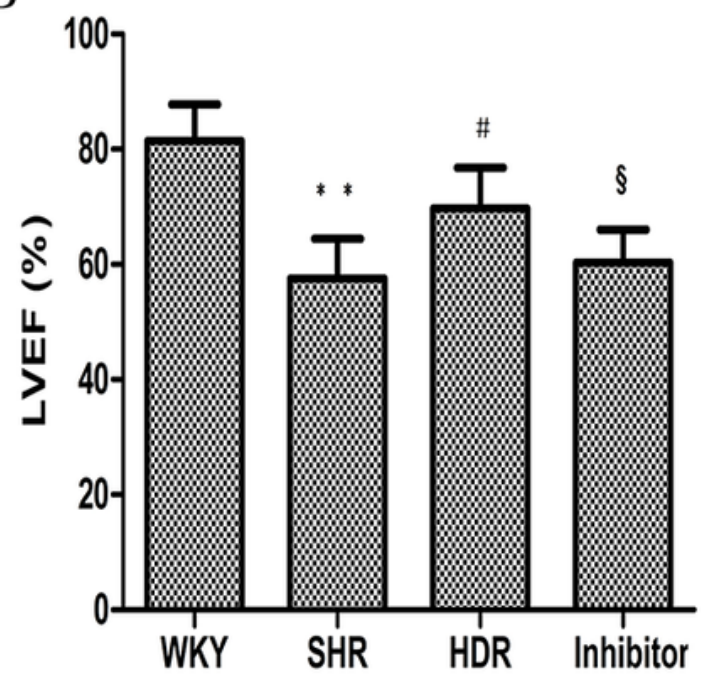

$\mathrm{C}$

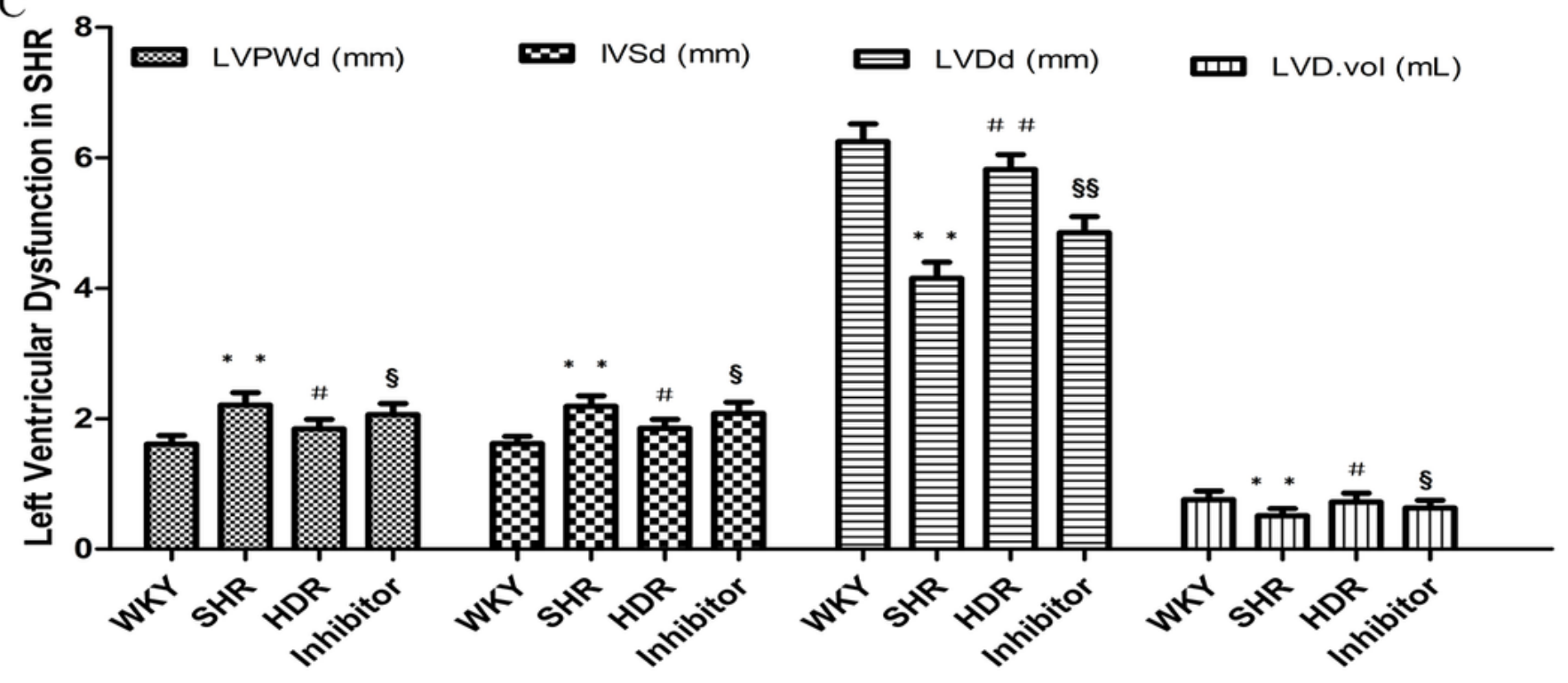

$\mathrm{D}$

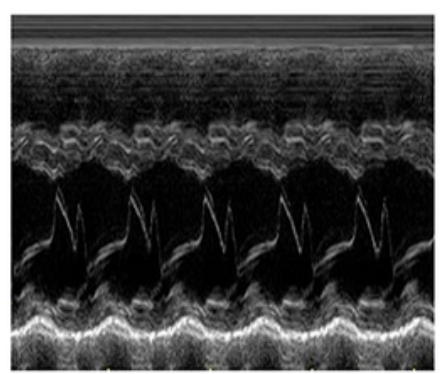

WKY

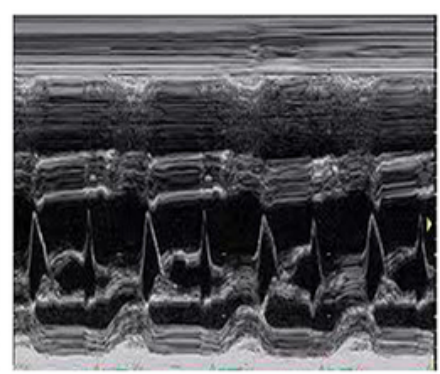

SHR

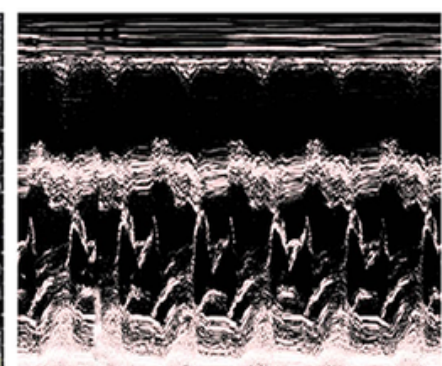

HDR

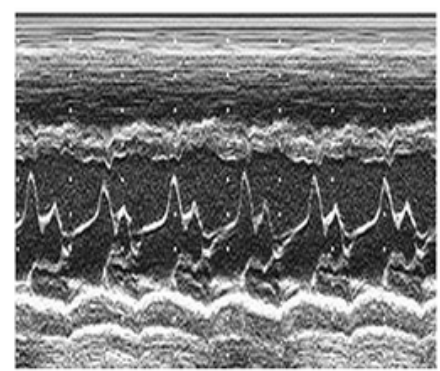

Inhibitor

Figure 1

The effect of HDR on blood pressure and cardiac function in rats with ventricular hypertrophy. A: Tail systolic blood pressure (SBP) and diastolic blood pressure (DBP) were reduced by HDR treatment. B: Functional parameters such as ejection fraction (EF, \%) were calculated using LVDd and LVDs. C: Interventricular septal thickness (IVSd), left ventricular posterior wall thickness (LVPWd), LV end-diastolic (LVDd) and end-systolic chamber dimensions. Left ventricle volume (LVD.vol) was measured from short- 
axis views of the LV under M-mode tracings. D: Examples of $\mathrm{M}$ mode echocardiographic recordings for WKY and SH rats, HDR and inhibitor-treated $\mathrm{SH}$ rats at 16 weeks age. ${ }^{*} \mathrm{P}<0.05,{ }^{\star} * \mathrm{P}<0.01$ compared with the WKY group; \# $\mathrm{P}<0.05$, \#\#P<0.01 compared with the SH group; $\S \mathrm{P}<0.05$, $\S \S \mathrm{P}<0.01$ compared with the HDR group.
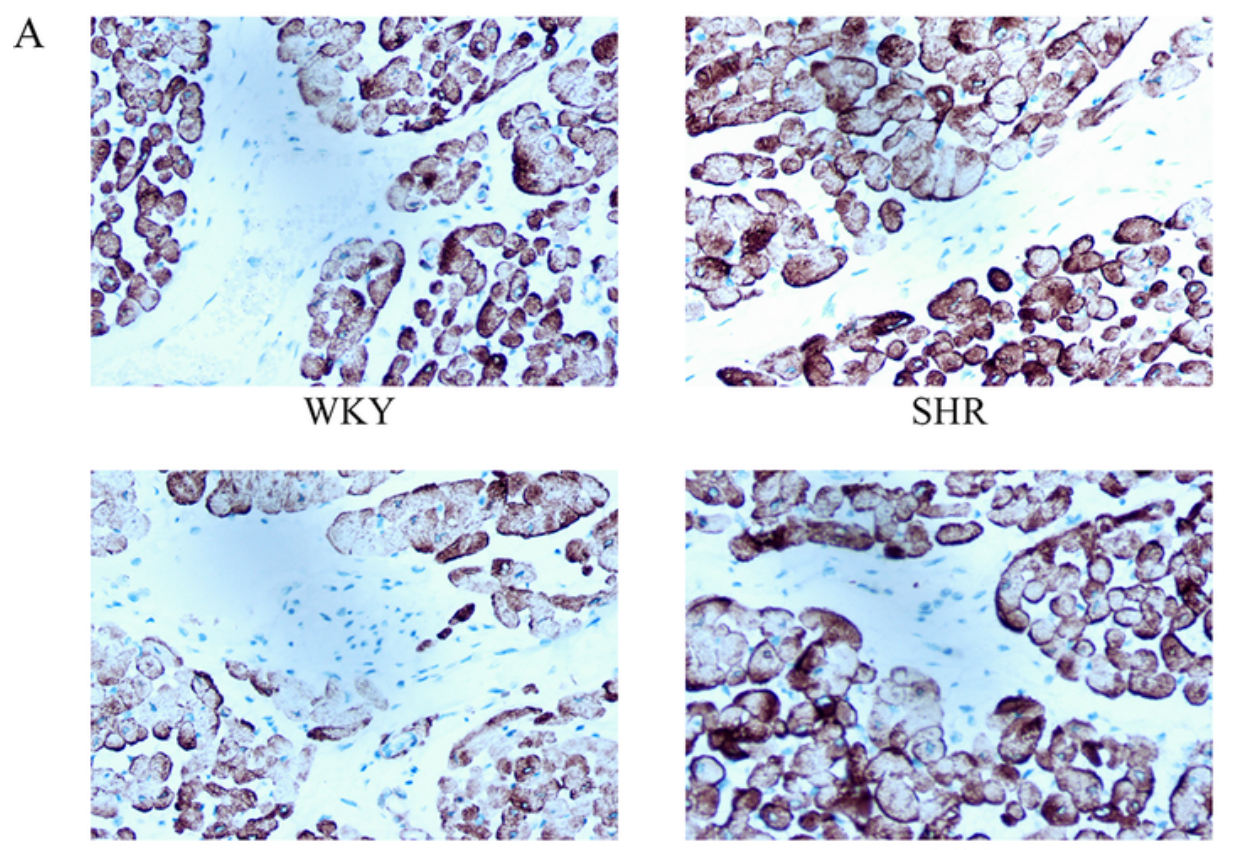

HDR

Inhibitor
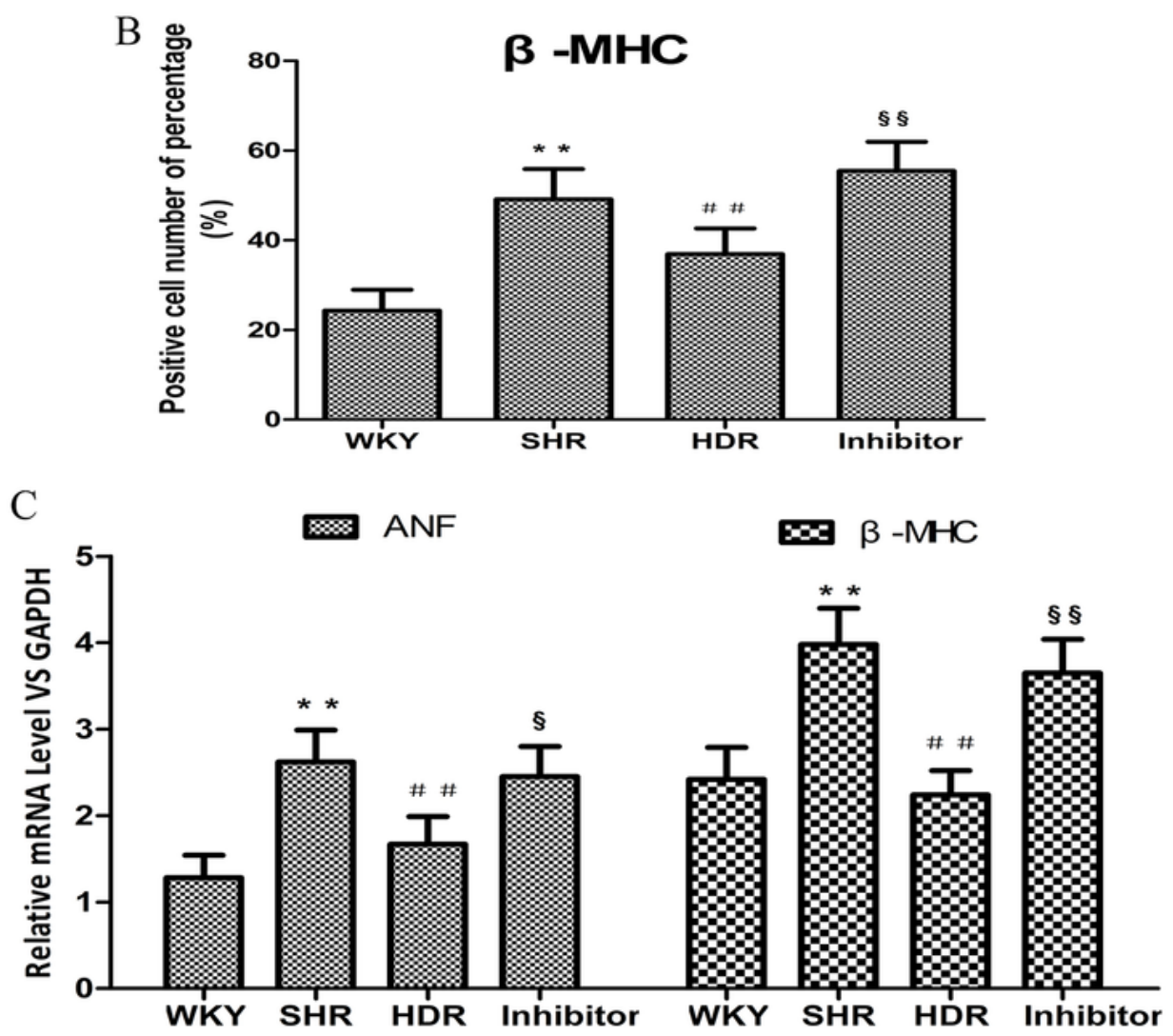

Figure 2 
The effect of HDR on the hypertrophic marker gene and protein expression in rats with ventricular hypertrophy. A: Immunohistochemical analysis of $\beta-\mathrm{MHC}$ in myocardial tissue (brown indicates positive cells, $\times 200)$. B: Semiquantitative rank scores for features of $\beta$-MHC-positive cells in myocardial tissue. C: Reverse transcription-quantitative polymerase chain reaction (RT-qPCR) was used to detect mRNA expression of ANF and $\beta-M H C$. ${ }^{*} P<0.05,{ }^{*} \mathrm{P}<0.01$ compared with the WKY group; \# $P<0.05$, \#\#P<0.01 compared with the SH group; $\S \mathrm{P}<0.05, \S \S \mathrm{P}<0.01$ compared with the HDR group.

A

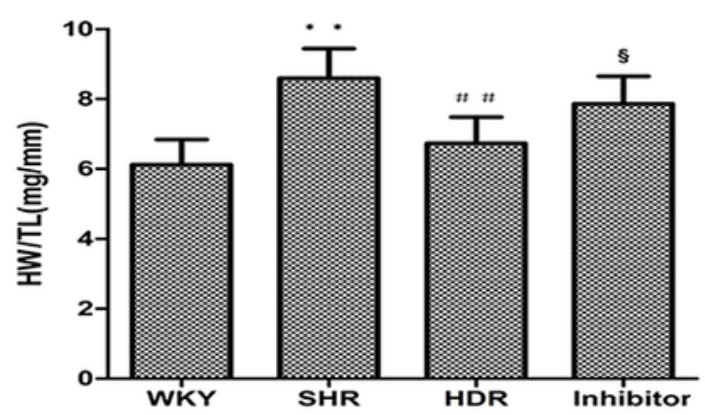

B

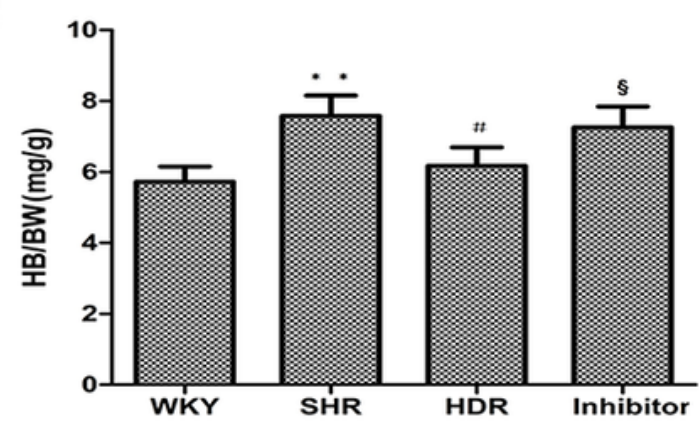

$\mathrm{C}$

$\mathrm{D}$

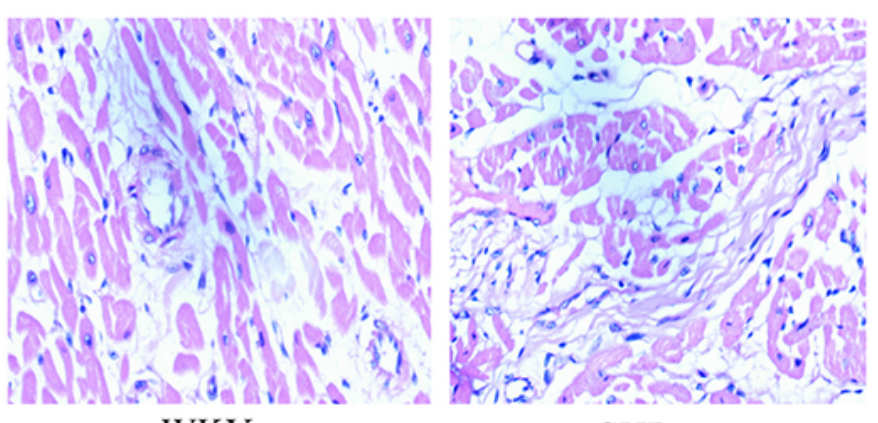
WKY

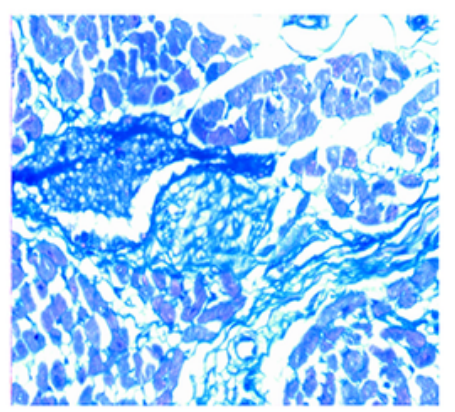
WKY

E

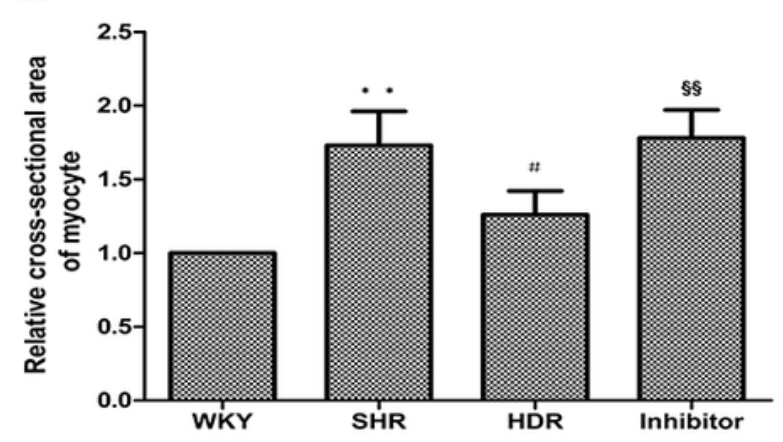

SHR

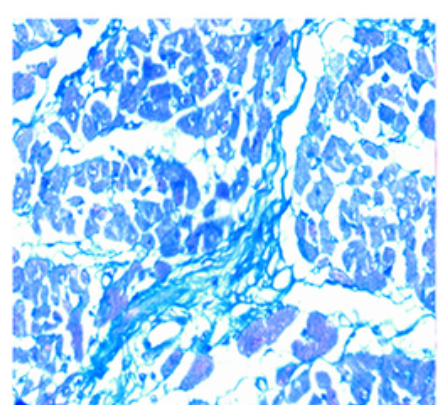

SHR
F

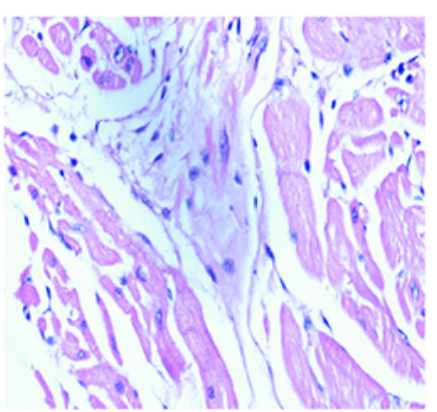

HDR

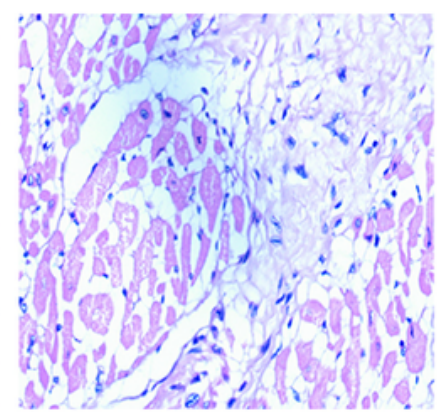

Inhibitor

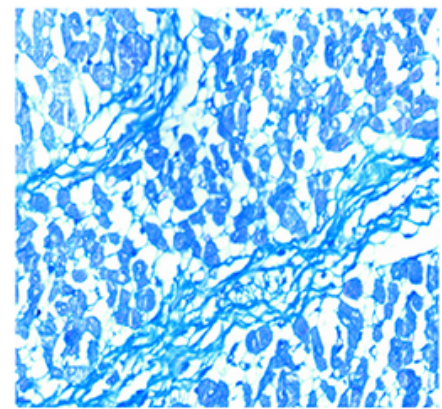

HDR

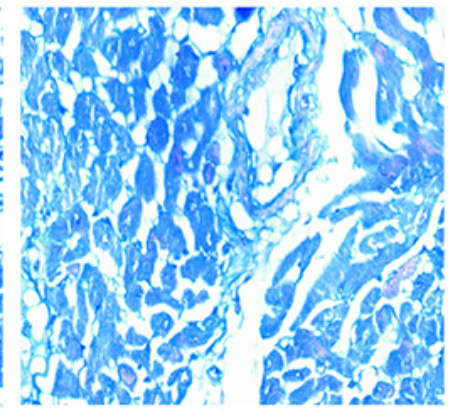

Inhibitor

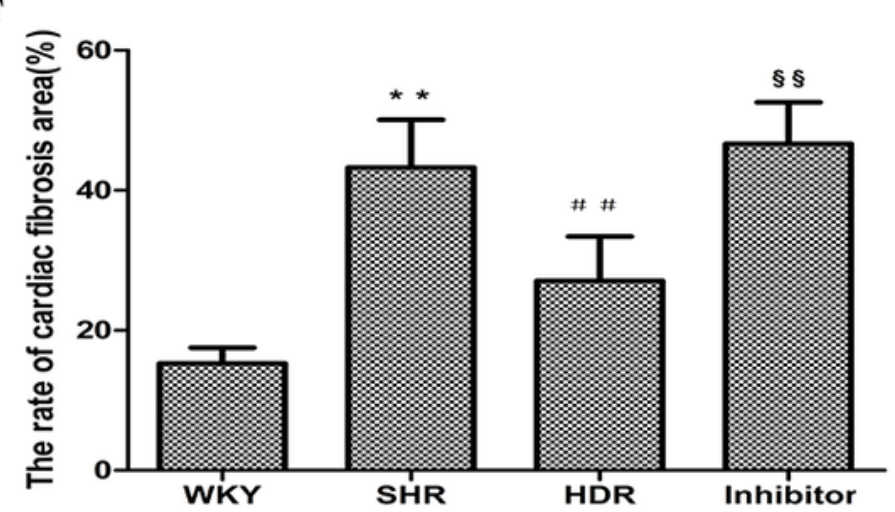

Figure 3 
The effect of HDR on heart histopathology in rats with cardiac remodeling. A: The ratios of heart weight to body weight (HW/BW). B: The heart weight to tibia length $(\mathrm{HW} / \mathrm{TL})$ in each group. C: Representative images of H\&E staining of heart tissues, $\times 400$; D: Representative images of Masson's trichrome staining of heart tissues, $\times 400$; $\mathrm{E}$ : The cross-sectional area of cardiomyocytes as indicated by HE staining. F: Quantification of myocardial interstitial and perivascular fibrotic area. ${ }^{*} P<0.05,{ }^{*} P<0.01$ compared with the WKY group; \# $\mathrm{P}<0.05$, \#\#P<0.01 compared with the SH group; $\S \mathrm{P}<0.05$, $\S \S \mathrm{P}<0.01$ compared with the HDR group.

A

WKY
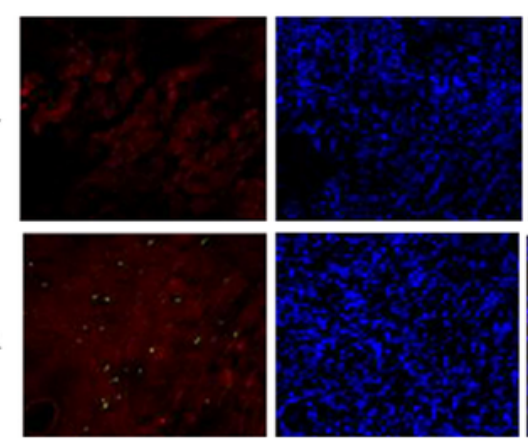

SHR
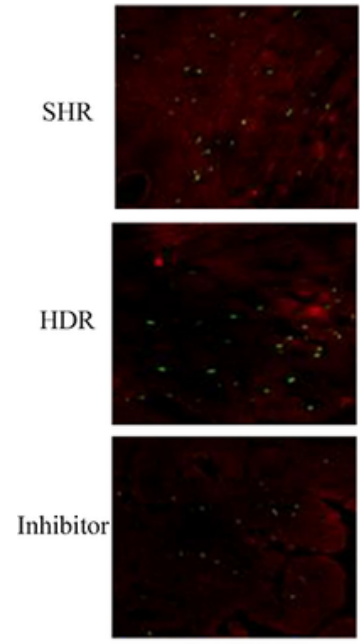

$\mathrm{cTnl} / \mathrm{TUNEL}$

$\mathrm{D}$

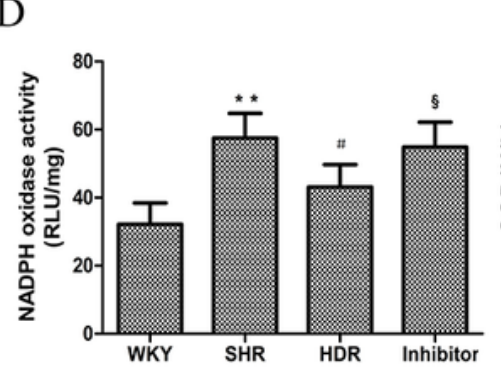

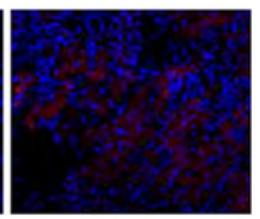

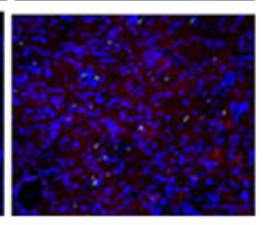

E

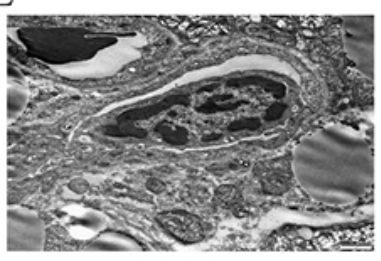

WKY

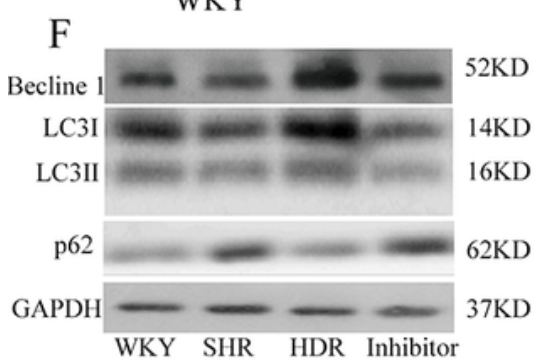

B
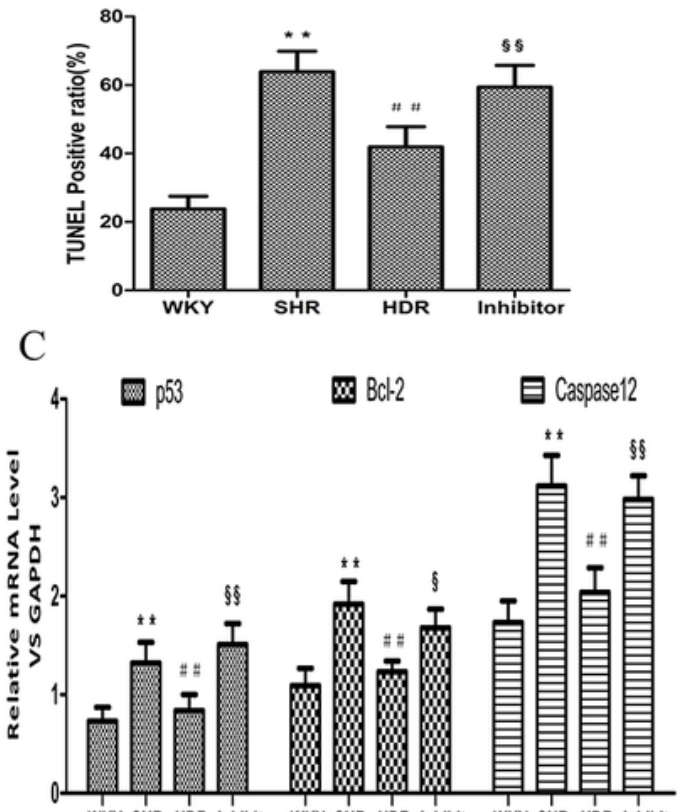

WKY SHR HDR Inhibitor WKY SHR HDR Inhibitor WKY SHR HDR Inhibitor

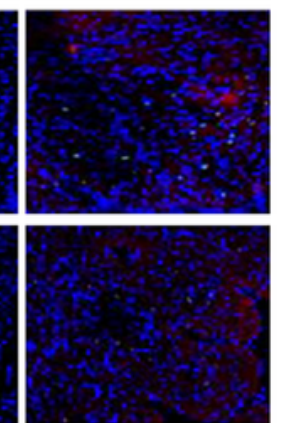

Merge
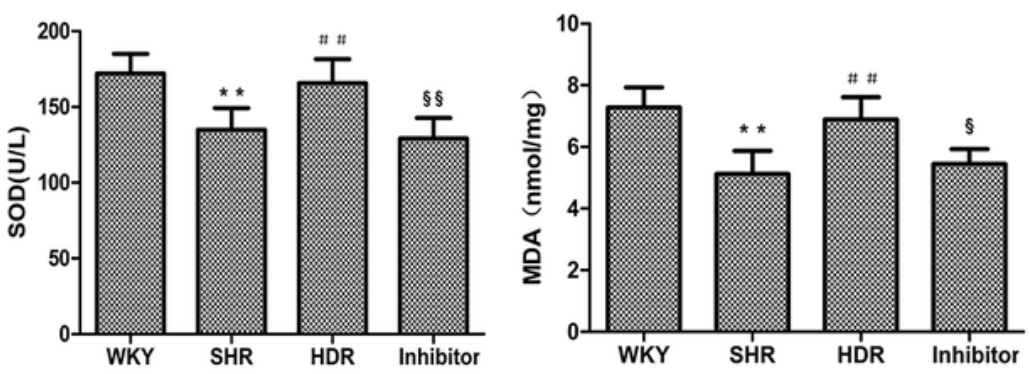

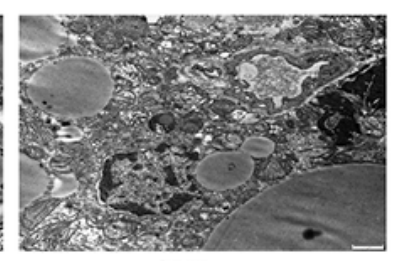

SHR

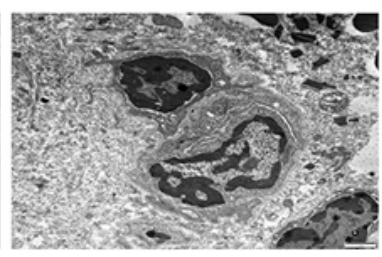

HDR

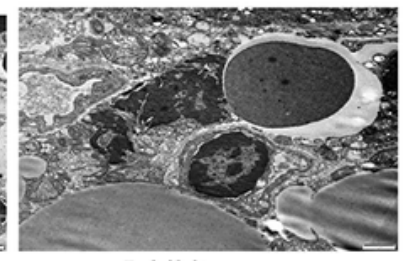

Inhibitor

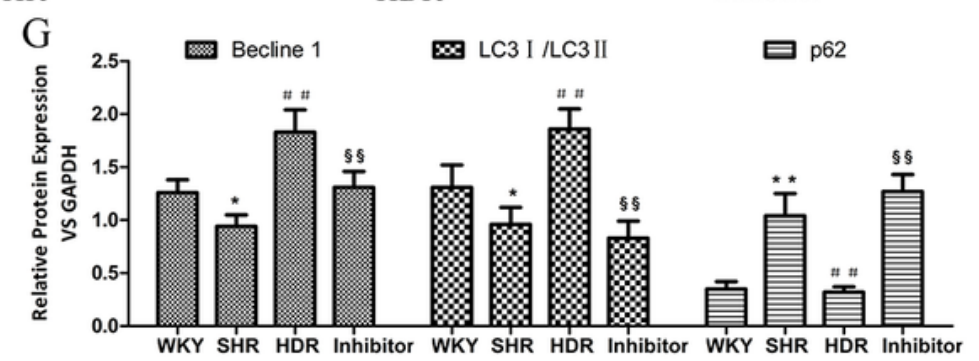




\section{Figure 4}

HDR treatment increased autophagy, prevented apoptosis and reversed oxidative stress. A:

Cardiomyocyte apoptosis detected by the terminal deoxynucleotidyl transferase-mediated dUTP nick end labeling (TUNEL) ( $\times 100$, apoptotic cells in red, cell nuclei in blue [DAPI]). B: Quantification of cardiomyocyte apoptosis-positive area. C: RT-qPCR was used to detect mRNA expression of apoptosisrelated markers, including in p53, Bcl-2 and Caspase 12. D: ELISA was used to detect the level of SOD and MDA in serum, and the activity of NADPH oxidase in heart tissue. E: Transmission electron microscopy was used to analyze the formation of autophagy in myocardial tissue $(\times 8000)$. F: Western blot analysis was used to examine expression of the autophagy-related proteins including p62, Beclin 1 and LC3区/LC3囚. G: Quantification of the relative protein levels of p62, Beclin 1 and LC3区/LC3囚. Data are presented as means $\pm S E M$, and $n=10$. HDR, HuoXue DiTan recipe. DAPI, 4',6-diamidino-2-phenylindole. ${ }^{*} \mathrm{P}<0.05$, ${ }^{\star *} \mathrm{P}<0.01$ compared with the WKY group; \# $\mathrm{P}<0.05$, \#\#P<0.01 compared with the SH group; $\S \mathrm{P}<0.05, \S \S \mathrm{P}<0.01$ compared with the HDR group. 


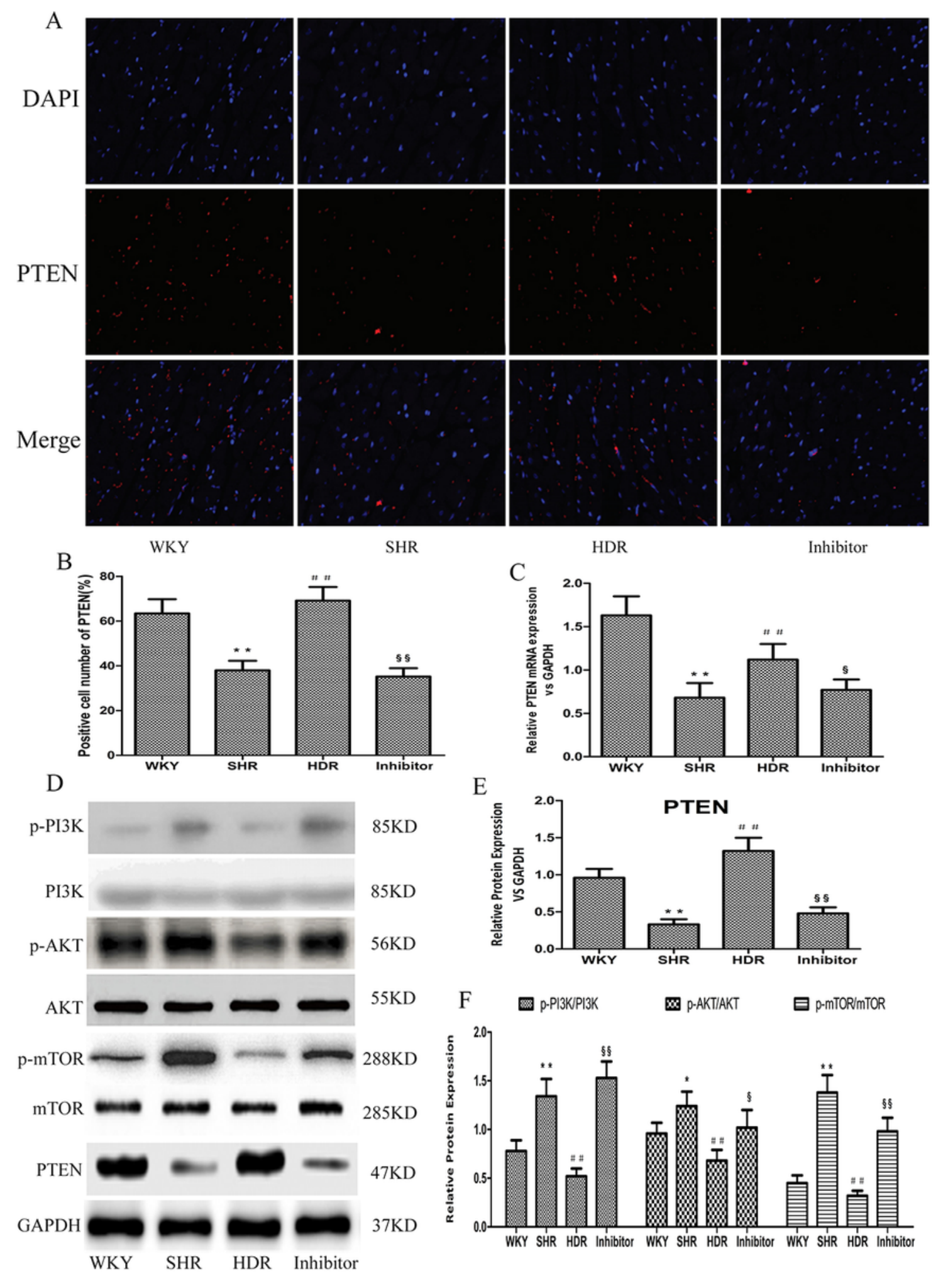

Figure 5

HDR increased autophagy and inhibited apoptosis in ventricular hypertrophy through the PTEN/PI3K/AKT/mTOR signal pathway. A: Immunofluorescence staining of PTEN in the myocardial tissues of each group of rats $(\times 100)$. B: Quantification of the PTEN-positive area. C: RT-qPCR was used to detect mRNA expression of PTEN. D: Western blot analysis was used to examine PTEN, PI3K, p-PI3K, AKT, p-AKT, mTOR and p-mTOR expression levels. E: Quantification of the relative protein levels of PTEN. F: 
Quantification of the relative protein levels of p-PI3K, p-AKT and p-mTOR. Data are presented as means \pm SEM, and $n=10$. PI3K, phosphatidylinositol 3-kinase; $p$-PI3K, phosphorylated-PI3K; AKT, protein kinase B; p-AKT, phosphorylated-AKT; mTOR, mammalian target of rapamycin; p-mTOR, phosphorylated-mTOR; HDR, HuoXue DiTan recipe. DAPI, 4',6-diamidino-2-phenylindole. ${ }^{*} P<0.05, * * P<0.01$ compared with the WKY group; \# $\mathrm{P}<0.05$, \#\#P<0.01 compared with the $\mathrm{SH}$ group; $\S \mathrm{P}<0.05, \S \S \mathrm{P}<0.01$ compared with the HDR group.

\section{Supplementary Files}

This is a list of supplementary files associated with this preprint. Click to download.

- S1.doc 